\title{
Corneal Cross-linking in Patients Younger than 18 Years: Long-term Follow-up in Three Israeli Medical Centers
}

\author{
${ }^{1}$ Erez Bakshi, ${ }^{2}$ Irina S Barequet, ${ }^{3}$ Isaac Aizenman, ${ }^{4}$ Samuel Levinger, ${ }^{5}$ Isaac Avni, ${ }^{6}$ David Zadok
}

\begin{abstract}
Purpose: To report refractive, topographic and safety outcomes of corneal cross-linking $(\mathrm{CXL})$ in patients younger than 18 years of age with progressive keratoconus.
\end{abstract}

Materials and methods: In this retrospective study, we enrolled 31 eyes of 21 children aged 11 to 17 years that underwent corneal riboflavin-ultraviolet $A$ induced $C X L$ due to progressive keratoconus at three different ophthalmology departments in Israel. They were followed for 3 to 48 months (average $23 \pm$ 13.6 months). Evaluated parameters were uncorrected visual acuity (UCVA), best spectacle corrected visual acuity (BSCVA), manifest refraction, pachymetry, slit- lamp examination and corneal topography at baseline and at 1,3,6,12,24 and 48 months.

Results: We found a nonsignificant improvement in UCVA and BSCVA with a small reduction of manifest cylinder and no significant change in spherical equivalent or K-values. Following CXL, stability of UCVA and BSCVA at the last follow-up examination was found in 71 and $77 \%$ of treated eyes, respectively. No permanent adverse events have been recorded throughout the study period.

Conclusion: In our series, CXL was a safe procedure in the pediatric population. Stabilization of progressive keratoconus was achieved in visual acuity, refractive and topography parameters with no improvement in corneal indices in contrary to adult CXL treatment.

Keywords: Progressive keratoconus, Collagen cross-linking, Riboflavin, UVA irradiation, Pediatric.

How to cite this article: Bakshi E, Barequet IS, Aizenman I, Levinger S, Avni I, Zadok D. Corneal Corss-linking in Patients

\footnotetext{
${ }^{1}$ Clinical Instructor, ${ }^{2}$ Senior Lecturer, ${ }^{3,4}$ Private Practitioner ${ }^{5,6}$ Professor

${ }^{1,5,6}$ Department of Ophthalmology, Assaf Harofeh Medical Center, Zerifin; Sackler Faculty of Medicine, Tel Aviv University Tel Aviv, Israel

${ }^{2}$ Department of Ophthalmology, Goldschleger Eye Institute Sheba Medical Center, Tel Hashomer; Sackler Faculty of Medicine, Tel Aviv University, Tel Aviv, Israel

${ }^{3,4}$ Private Practice, 'Enaim' Medical Center, Jerusalem and Tel Aviv, Israel

Corresponding Author: Erez Bakshi, Clinical Instructor Department of Ophthalmology, Assaf Harofeh Medical Center Zerifin-73000, Israel, Phone: +972-505191580, Fax: +97289779357, e-mail: erezbakshi@gmail.com
}

Note: The first two authors contributed equally to this paper
Younger than 18 Years: Long-term Follow-up in Three Israeli Medical Centers. Int J Kerat Ect Cor Dis 2014;3(2):84-87.

\section{Source of support: Nil}

Conflict of interest: None declared

\section{INTRODUCTION}

Keratoconus is a progressive noninflammatory disorder, characterized by corneal ectasia and thinning due to changes in collagen structure and biomechanical stromal weakening. Progression of this degenerative process is manifested by increasing corneal distortion, irregular astigmatism, progressive myopia and central corneal scarring, resulting in visual acuity deterioration. ${ }^{1-4}$

Mild cases may require spectacles or rigid permeable contact lenses, and in recent years also wavefront-corrected spectacles and soft contact lenses. ${ }^{5}$ Mild to moderate keratoconus with unsatisfactory best-corrected visual acuity (BCVA) or contact lens intolerance may necessitate surgical intervention such as intracorneal ring segments implantation; however long-term follow-up demonstrates that the resulting refractive improvement is only temporary, and a significant progression of keratometry values in operated eyes has been reported. ${ }^{6,7}$ In cases of further progression of the ectasia and failure of the above mentioned tools, corneal transplantation is the treatment of choice in up to $20 \%$ of patients. ${ }^{8,9}$

A decade ago, Wollensak and associated have introduced a novel minimally invasive technique using riboflavin $\backslash U V A$ corneal collagen cross-linking (CXL), which has been shown to enhance corneal biomechanical stiffening, stabilize and even arrest progression of keratoconus in adult eyes, with no permanent deleterious effects, thus rendering corneal transplantation unnecessary in some patients. ${ }^{10-18}$

It seems that keratoconus has a tendency to progress faster in children than in adults, and therefore the consequence of unsatisfactory visual acuity or contact lens intolerance may be encountered sooner. Vinciguerra and associates have recently reported their results of a 2 years follow-up after corneal CXL in patients younger than 18 years with documented progressive keratoconus. ${ }^{19}$ According to their prospective study, CXL improved uncorrected and best spectacle corrected visual acuity (BSCVA) in the study patients, an improvement that was found to be statistically significant. However, in our previous study on pediatric keratoconus, 
the results were less conclusive, and apart from achieving stability in most patients, no statistically significant improvement was noted in any parameter. ${ }^{20}$

Therefore, in this study, we retrospectively analyzed visual acuity, refractive and topographic outcomes in a larger group of patients (31 eyes in comparison to 9 eyes in our previous study) younger than 18 years with a documented progressive keratoconus in three different and independent medical centers in Israel.

\section{MATERIALS AND METHODS}

\section{Patients}

Thirty-one eyes of 21 patients in which keratoconus progression was documented in the preceding 6 months were enrolled to this retrospective study at 3 ophthalmology departments in Israel: Assaf Harofeh Medical Center, Sheba Medical Center and 'Enaim' Medical Center, during 2009-2013.

Inclusion criteria included: age younger than 18 years at the time of the procedure, progressive keratoconus and corneal thickness of at least $400 \mu \mathrm{m}$. Progression was defined as 1 or more of the following changes over a period of 6 months: an increase of at least 1.00 diopter (D) in $\mathrm{K}_{\max }$, an increase of at least $1.00 \mathrm{D}$ in manifest cylinder, an increase of at least $0.50 \mathrm{D}$ in manifest refraction spherical equivalent (SE), loss of BSCVA of more than 2 lines or a subjective decrease in visual acuity (VA).

Exclusion criteria included: corneal thickness less than $400 \mu \mathrm{m}$ at the thinnest point, severe surface disease, a history of herpetic keratitis, a previous ocular surgery, concurrent corneal infection, presence of central or paracentral corneal opacities or a documented autoimmune disease.

Distance UCVA and BSCVA (in log MAR) as well as manifest refraction were assessed using the Snellen chart preoperatively and 1, 3, 6, 12 and 24 months postoperatively. The refraction was meticulously evaluated by the operating surgeon. Topography measurements were obtained preoperatively and 1, 3, 6, 12 and 24 months postoperatively using a rotating Scheimpflug camera (Pentacam, Oculus, Inc.) or the TMS 4 topographic modeling system (TOMEY, Japan). Recorded parameters were maximum, minimum and average $\mathrm{K}$ values $\left(\mathrm{K}_{\max }, \mathrm{K}_{\min }\right.$ and $\mathrm{K}_{\text {ave }}$ respectively), corneal astigmatism (simulated $\mathrm{K}$ ) and corneal thickness.

\section{Surgical Technique}

Each one of the procedures were performed by four surgeons (DZ, IB, IA and SL), using the 'Dresden protocol' as previously published. ${ }^{9}$ The surgical procedure was performed under topical anesthesia preceded by standard prepping and draping. The central zone of an area $8 \mathrm{~mm}$ in diameter corneal epithelium was removed using a blunt metal spatula. Drops of riboflavin $0.1 \%$ combined with dextran $20 \%$ were instilled every 2 minutes for 30 minutes. After achieving a strong yellow flare in the anterior chamber, the cornea was exposed to UV-A when the patient is in recumbent position. The patient was instructed to focus on the central LED of the probe and the surgeon also confirmed centration in order to achieve maximal stability throughout the procedure. The UV-A (365-370 nm) light was introduced via UVA source located $5 \mathrm{~cm}$ from the eye (UVX produced by IROC) with a light intensity of $3 \mathrm{~mW} / \mathrm{cm}^{2}$. Riboflavin eye drops continued to be administered every 2 minutes during the UV-A treatment. At the conclusion of eth procedure a bandage contact lens was applied and maintained until the re-epithelialization was complete. The postoperative treatment included topical Oflox (ofloxacin 0.3\%) drops for 3 days, and FML (fluorometholone $0.1 \%$ ) for 3 weeks.

\section{Statistical Analysis}

Statistical analysis was performed with SPSS software using (version 16, SPSS Inc) and the data was analyzed using the 2-tailed t-test for each parameter. Data is expressed as mean differences between pre and post for each parameter.

\section{RESULTS}

Thirty-one consecutive eyes of 21 patients were identified. All patients had CXL and had a follow-up period of 3 to 48 months (average, $23 \pm 13.6$ months). Twenty-five eyes (80\%) completed at least 12 months of follow-up. All patients were males, and the mean age was $14.7 \pm 1.6$ years (11-17 years). Neither intraoperative nor postoperative complications were encountered, with the exception of one case of corneal haze in that resolved completely with topical steroids treatment.

Graphs 1 and 2 contain data concerning uncorrected and best spectacle corrected visual acuity, respectively, expressed in logarithm of minimal angle of resolution units. There was a slight improvement in UCVA that was statistically significant $(0.74 \pm 0.3$ preoperatively and $0.64 \pm 0.33$ postoperatively, $\mathrm{p}=0.039)$ and a modest statistically insignificant improvement in BSCVA $(0.29 \pm 0.22$ preoperatively and $0.26 \pm 0.17$ postoperatively, $\mathrm{p}=0.22$ ). Visual acuity was evaluated at each encounter, as was elaborated earlier, but the data shown on the graphs express visual acuity at baseline and at the final encounter. Most of the patients achieved a long-term stability or improvement in both UCVA ( 22 of 31 eyes, $71 \%$ ) and BSCVA (24 of 31 eyes, $77 \%$ ). There was a nonstatistically significant reduction in manifest cylinder, from $4.05 \mathrm{D}$ preoperatively to $4.00 \mathrm{D}$ postoperatively, $(p=0.91)$, and no statistically significant change in the spherical equivalent ( $-1.19 \mathrm{D}$ pre- and 1.22 $\mathrm{D}$ postoperatively, $\mathrm{p}=0.91$ ). 


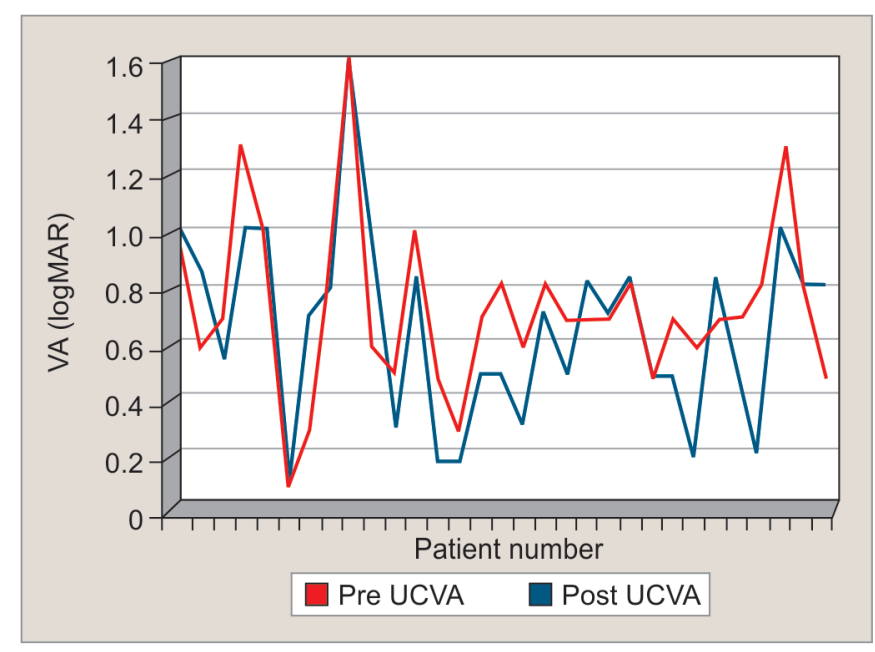

Graph 1: Uncorrected visual acuity pre- and postoperatively

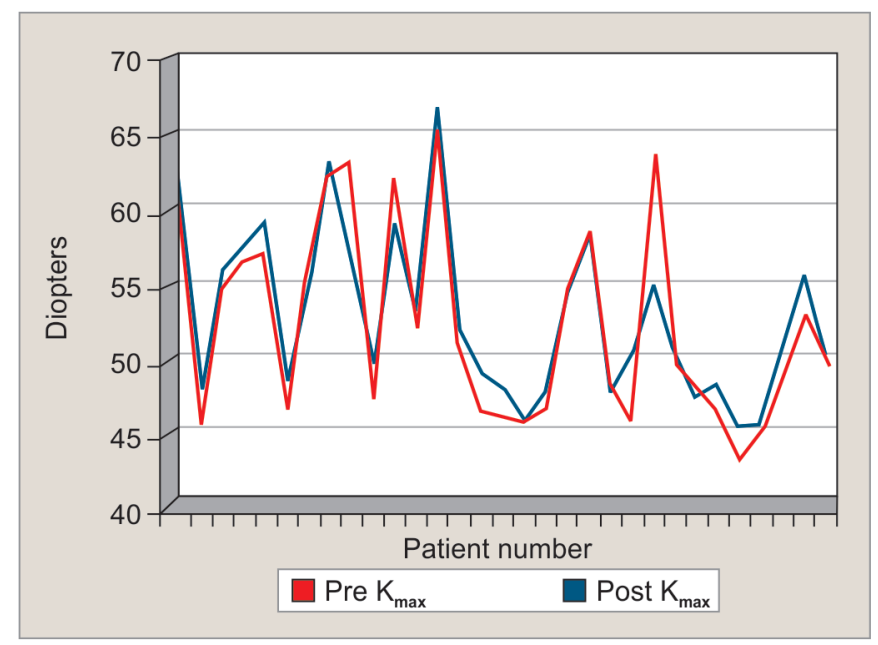

Graph 3: $\mathrm{K}_{\max }$ pre- and postoperatively

There was no statistically significant change in average $\mathrm{K}_{\max }(52.55 \mathrm{D}$ pre- and 52.71D postoperatively, Graph 3), in $\mathrm{K}_{\min }\left(45.7 \mathrm{D}\right.$ pre- and $46.1 \mathrm{D}$ postoperatively) or in $\mathrm{K}_{\text {ave }}$ (48.7D pre- and 49.7D postoperatively).

\section{DISCUSSION}

Our results showed that CXL stabilized visual acuity, refractive and topography parameters in a pediatric population with progressive keratoconus.

In the last decade, corneal CXL with riboflavin and UV-A has become an important tool aiming stabilize the cornea in patients with keratoconus. This minimally invasive technique has been shown in several studies to be a safe and effective mean, being able to halt the progression of keratoconus, and thus preserve visual function, and even reduce the need for keratoplasty in some patients. ${ }^{10,21-24}$ CXL performed in patients with documented progression of keratoconus it showed long-lasting stability of at least 24 months, and in some cases even improved visual acuity and corneal topographic indices with. ${ }^{25-27}$

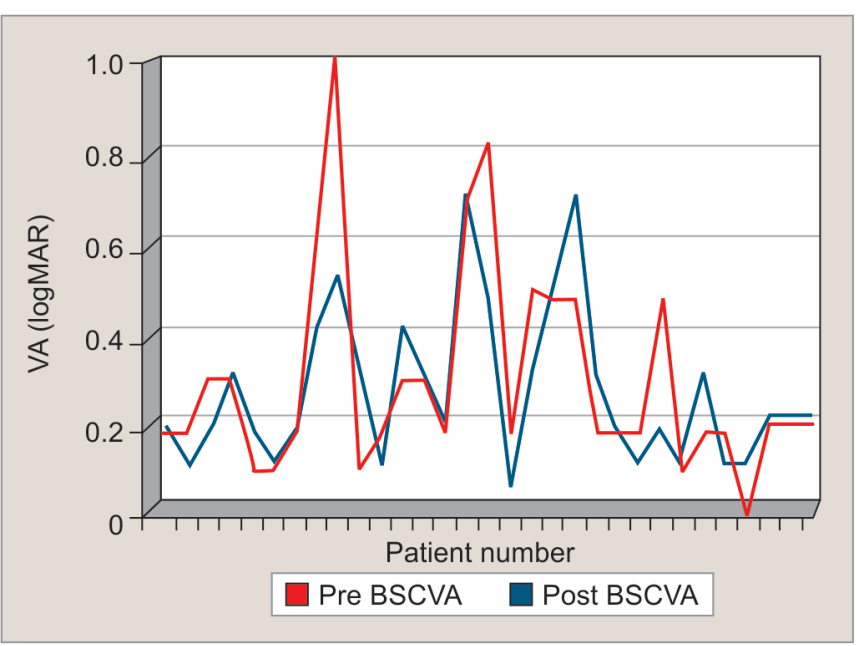

Graph 2: Best spectacle-corrected visual acuity pre- and postoperatively

Keratoconus is often more advanced in children than in adults, with faster disease progression. Early detection and close monitoring are therefore crucial in young patients. ${ }^{28}$ Therefore, CXL may play an even more important role in this high-risk population. Only a few studies reported CXL in pediatric keratoconus. Soeters et $\mathrm{al}^{29}$ reported regression or at least stabilization of the $\mathrm{K}_{\max }$ and BCVA following CXL in 5 eyes of 4 children with rapidly progressive keratoconus. Zotta et $\mathrm{al}^{30}$ reported stabilization of keratometric indices in 8 eyes of 4 children who underwent CXL for progressive keratoconus. Arora et $\mathrm{al}^{31}$ showed improvement in visual acuity and keratometric data in 15 eyes of pediatric patients with moderate keratoconus. Vinciguerra et al ${ }^{19}$ recently published a larger prospective study on CXL in 40 eyes of 40 patients younger than 18 years and reported a statistically significant $(\mathrm{p}<0.05)$ improvement in UCVA $(0.79 \pm 0.21$ at baseline and $0.58 \pm 0.18$ two years after the procedure) and $\operatorname{BSCVA}(0.39 \pm 0.10$ at baseline and $0.20 \pm 0.09$ two years after the procedure). They also reported a decrease in the value of spherical equivalent by $1.57 \mathrm{D}$ at 24 months with a concomitant improvement in all keratometric parameters. Although our study found visual acuity stability (improvement or no worsening) in most patients, only a slight and insignificant improvement UCVA and BSCVA was observed, with no change in corneal indices. We believe that these differences in outcomes could be related to a difference in patient selection between our study and Vinciguerra's study: keratoconus progression in the latter study was defined as a change in either myopia or astigmatism of at least 3 diopters in the previous 3 months, or a mean central $\mathrm{K}$ reading change of at least $1.5 \mathrm{D}$ observed in 2 consecutive topographies during the preceding 3 months. ${ }^{19}$ On the other hand, in our study progression was defined as an increase of at least $1.00 \mathrm{D}$ in $\mathrm{K}_{\max }$, an increase of at least 1.00D in manifest cylinder, an increase of at least $0.50 \mathrm{D}$ in manifest refraction spherical 
equivalent (SE), loss of BSCVA of more than 2 lines or a subjective decrease in VA. Thus, the progression rate in Vinciguerra's study was significantly higher than in ours, meaning that progression in the former study was a definite fact and keratoconus was indeed rapidly progressing, while in this study we enrolled patients with a relatively modest progression. If that is the case, it may be cautiously assumed, that corneal cross-linking should still remain as an option to halt progression in younge $r$ patients, but perhaps progression criteria should be more meticulously determined.

The limitations of our study include the retrospective method of retrieval of the data performed in 3 centers, however using the same methods.

\section{CONCLUSION}

Our data support the safely of CXL in pediatric patients, which is capable of stabilizing visual acuity, refraction and corneal indices. Further larger scale prospective studies are required to determine the optimal indication criteria for CXL in children with keratoconus and to better predict the improvement in visual parameters and not only stabilization and preservation of the given status.

\section{REFERENCES}

1. Rabinowitz YS. Keratoconus. Surv Ophthalmol 1998;42: 297-319.

2. Tuori AJ, Virtanen I, Aine E, et al. The immunohistochemical composition of corneal basement membrane in keratoconus. Curr Eye Res 1997;16:792-801.

3. Cheng EL, Maruyama I, Sundar Raj N, et al. Expression of type XII collagen and hemidesmosome-associated proteins in keratoconus corneas. Curr Eye Res 2001;22:333-340.

4. Goldich Y, Marcovich AL, Barkana Y, et al. Clinical and corneal biomechanical changes after collagen cross-linking with riboflavin and UV irradiation in patients with progressive keratoconus: results after 2 years of follow-up. Cornea 2012 Jun;31(6): 609-614.

5. Marsack JD, Parker KE, Niu Y, et al. On-eye performance of custom wavefront-guided soft contact lenses in a habitual soft lens-wearing keratoconic patient. J Refract Surg 2007;23: 960-964.

6. Zare MA, Hashemi H, Salari MR. Intracorneal ring segment implantation for the management of keratoconus: safety and efficacy. J Cataract Refract Surg 2007;33:1886-1891.

7. Alió JL, Shabayek MH, Artola A. Intracorneal ring segments for keratoconus correction: long-term follow-up. J Cataract Refract Surg 2006;32:978-985.

8. Troutman RC, Lawless MA. Penetrating keratoplasty for keratoconus. Cornea 1987;6:298-305.

9. Lim L, Pesudovs K, Coster DJ. Penetrating keratoplasty for keratoconus: visual outcome and success. Ophthalmology 2000; 107:1125-1131.

10. Wollensak G, Spoerl E, Seiler T. Riboflavin/ultraviolet: A induced collagen cross-linking for the treatment of keratoconus. Am J Ophthalmol 2003;135:620-627.
11. Wollensak G, Spörl E, Seiler T. Treatment of keratoconus by collagen cross-linking. Ophthalmology 2003;100:44-49.

12. Wollensak G. Cross-linking treatment of progressive keratoconus: new hope. Curr Opin Ophthalmol 2006;17:356-60.

13. Wollensak G, Spoerl E, Seiler T. Stress-strain measurements of human and porcine corneas after riboflavin/ultravioletA induced collagen cross-linking. J Cataract Refract Surg 2003;29: 1780-1785.

14. Kohlhaas M, Spoerl E, Schilde T, et al. Biomechanical evidence of the distribution of cross-links in corneas treated with riboflavin and ultraviolet A light. J Cataract Refract Surg 2006;32:279-283.

15. Spoerl E, Huhle M, Seiler T. Induction of cross-links in corneal tissue. Exp Eye Res 1998;66:97-103.

16. Spoerl E, Seiler T. Techniques for stiffening the cornea. J Refract Surg 1999;15:711-713.

17. Spoerl E, Wollensak G, Seiler T. Increased resistance of crosslinked cornea against enzymatic digestion. Curr Eye Res 2004; 29:35-40.

18. Schilde T, Kohlhaas M, Spoerl E, et al. Enzymatic evidence of the depth dependence of stiffening on riboflavin/UVA treated corneas. Ophthalmology 2008;105:165-169.

19. Vinciguerra P, Albé E, Frueh BE, et al. Two-year corneal crosslinking results in patients younger than 18 years with documented progressive keratoconus. Am J Ophthalmol 2012;154:520-526.

20. Bakshi E, Barkana Y, Goldich Y, et al. Corneal corss-linking for progressive keratoconus in children - our experience. International $\mathrm{J}$ of Keratoconus and ecstatic corneal diseases 2012; 1:53-56.

21. Caporossi A, Baiocchi S, Mazzotta C, et al. Parasurgical therapy for keratoconus by riboflavin-ultraviolet type A induced crosslinking of corneal collagen: preliminary refractive results in an Italian study. J Cataract Refract Surg 2006;32:837-845.

22. Mazzotta C, Traversi C, Baiocchi S, et al. Corneal healing after riboflavin-ultraviolet $\mathrm{A}$ cross-linking determined by confocal laser scanning microscopy in vivo: early and late modifications Am J Ophthalmol 2008;146:527-533.

23. Spoerl E, Mrochen M, Sliney D, et al. Safety of UVA-riboflavin cross-linking of the corneal. Cornea 2007;26:385-389.

24. Goldich Y, Marcovich AL, Barkana Y, et al. Safety of corneal collagen cross-linking with UV-A and riboflavin in progressive keratoconus. Cornea 2010 Apr;29(4):409-411.

25. Greenstein SA, Fry KL, Hersh PS. Corneal topography indices after corneal collagen corss-linking for keratoconus and corneal ectasia: One-year results. J Cataract Refract Surg 2011;37:1282-1290.

26. Caporossi A, Mazzotta C, Baiocchi S, et al. Long-term results of riboflavin ultraviolet A corneal collagen cross-linking for keratoconus in Italy: the siena eye cross study. Am J Ophthalmol 2010;149:585-593.

27. Raiskup-Wolf F, Hoyer A, Spoerl E, et al. Collagen corss-linking with riboflavin and ultraviolet A light in keratoconus: long-term results. J Cataract Refract Surg 2008;34:796-801.

28. Léoni-Mesplié S, Mortemousque B, Touboul D, et al. Scalability and severity of keratoconus in children. Am J Ophthalmol 2012; 154:56-62.

29. Soeters N, Van der Lelij A, Van der Valk R, et al. Corneal crosslinking for progressive keratoconus in four children. J Pediatr Ophthalmol Strabismus 2011;48:e26-e29.

30. Zotta PG, Moschou KA, Diakonis VF, et al. Corneal collagen cross-linking for progressive keratoconus in pediatric patients: a feasibility study. J Refract Surg 2012;28:793-739.

31. Arora R, Gupta D, Goyal JL, et al. Results of corneal collagen cross-linking in pediatric patients. J Refract Surg 2012;28: 759-762. 